\title{
21. PALEOMAGNETISM OF LEG 37 BASALTS
}

\author{
E.R. Deutsch, R.R. Pätzold, and G.S. Murthy, Geomagnetic Research Laboratory, \\ Physics Department, Memorial University of Newfoundland, St. John's, Canada
}

\begin{abstract}
Paleomagnetic data on 46 samples from DSDP Leg 37 are reported. All samples from Site 335 have a reverse remanence with consistently steep inclinations even after alternating-field and thermal demagnetization. In a 275 -meter section of Hole $332 \mathrm{~B}$, the natural remanence vectors vary considerably between shallow positive and shallow negative inclinations, suggesting that the vertical component of remanence over this basalt section is insufficient to account for observed surface anomalies.
\end{abstract}

\section{EXPERIMENTS}

We have made magnetic measurements on 46 basalt cores from Hole 332B and Sites 334 and 335 of Leg 37: Natural remanence (NRM); alternating field demagnetizations; stepwise and continuous thermal demagnetizations; low-field susceptibility $(k)$; and intensity $\left(J_{t}\right)$ of thermoremanence (TRM). Data for 45 samples $^{1}$ are given in Chapters 2, 4, and 5 (this volume). The experimental scheme, apparatus, and techniques used are described in Deutsch and Pätzold (1976). Despite the absence of an azimuthal core orientation, some important paleomagnetic clues can be obtained by comparing the magnitude and polarity of paleoinclination $(I)$ for different depths in the same drill hole and between neighboring holes.

\section{NRM AND SUSCEPTIBILITY}

In 25 out of 45 samples stored for 3 months in a laboratory field of about 0.5 oe, storage resulted in less than $3^{\circ}$ angular shift of the NRM and the largest change was $11^{\circ}$ (Sample 332B-35-2, 33-36 cm). Most of the samples show large $Q_{n}$ values in the range 10-100 (Table 1) which are common in oceanic basalts and usually indicate the predominance of stable over viscous (VRM) components; this justifies the assumption of a negligible induced component usually made when interpreting sea-floor magnetic anomalies in terms of the Vine-Matthews hypothesis.

For each drill hole, Table 1 shows the ranges of measured $J_{n}, k$, and $Q_{n}$ along with their geometric mean values. All nine mean values fall within the ranges of $J_{n}$, $k$, or $Q_{n}$ values given by Lowrie (1974) for basalts from 26 previous DSDP sites. Our three mean intensity values $(J n)$ are somewhat larger than the overall mean $\left(J_{n}=2.06 \times 10^{-3}\right.$ Gauss $)$ of these 26 DSDP sites.

\footnotetext{
'Sample 332B-42-1, 13-15 cm was irregularly shaped and could not be accommodated in the spinner magnetometer.
}

\section{HOLE 332B}

It was found that negative (NRM) inclinations (Chapter 2, this volume), expected from the negative anomaly over the drill site, occur in only 14 of our 27 samples, with no obvious correlation between NRM polarity and depth. All inclinations are in the range $-39^{\circ}$ to $+33^{\circ}$, compared with the present inclination $\left( \pm 56^{\circ}\right)$ of an axial dipole field.

This raises some questions: (1) Assuming that negative inclinations originated in a reverse field at the ridge, do positive inclinations represent a secondary component, perhaps acquired during the present (Brunhes) normal-polarity epoch? Could the magnetism be self-reversed? (2) Alternatively, if negative and positive NRMs in different sampies are both primary, do the data represent a sampling of blocks that were magnetized near the ridge during successive opposite polarity intervals (or during a polarity transition) and became mechanically mixed on traveling to the drill site? Or does the positive remanence reside in normally magnetized intrusive rocks (JOIDES Scientific Staff, Leg 37, 1974)? (3) Why are all NRMs systematically shallower than the earth's field at the site?

Demagnetization results (Tables 2, 3A, 3B; Figures I, $2,3)$ do not readily answer these questions: Sample $332 \mathrm{~B}-35-2,33-36 \mathrm{~cm}$ carried a VRM removable at 150 oe, and in higher fields the direction stabilized at I $\sim-42^{\circ}$ compared to $+6^{\circ}$ for NRM. However, Sample $332 \mathrm{~B}-36-2,59-61 \mathrm{~cm}$ (Figure 1) was directionally stable to 300 oe, and at higher steps its inclination remained close to the NRM value of $+26^{\circ}$. Both stepwise and continuous thermal demagnetizations (Tables 3A, 3B; Figures 2, 3) showed the natural remanent magnetization to be very stable up to $550^{\circ} \mathrm{C}$, indicating that the shallow NRM inclinations of Hole 332B are indeed of primary remanence.

Omitting obvious VRM cases, it is difficult to reconcile these results with the presence in positive NRMs of any except very hard secondary components (Question 
TABLE 1

Summary of $J_{n}, k$, and $Q_{n}$ Data

\begin{tabular}{lrllcccc}
\hline & & \multicolumn{3}{c}{$\begin{array}{c}\text { Range of } \\
k \times 10^{3}\end{array}$} & \multicolumn{3}{c}{ Average } \\
Hole & $\mathrm{N}$ & $\begin{array}{c}J_{n \times 10^{3}} \\
\text { (Gauss) }\end{array}$ & $\begin{array}{c}k \times 1 \mathrm{oe}) \\
\text { (G/2) }\end{array}$ & $Q_{n}$ & $\begin{array}{c}J_{n} \times 10^{3} \\
\text { (Gauss) }\end{array}$ & $\begin{array}{c}k \times 10^{3} \\
\text { (G/oe) }\end{array}$ & $Q_{n}$ \\
\hline $332 \mathrm{~B}$ & 27 & $0.743-19.6$ & $0.125-1.67$ & $2.1-126$ & 3.08 & 0.280 & 24 \\
334 & 2 & $1.54-2.94$ & $0.307-4.22$ & $1.5-11$ & 2.13 & 1.14 & 4.1 \\
335 & 16 & $1.90-10.1$ & $0.088-0.259$ & $21-163$ & 3.78 & 0.175 & 48 \\
\hline
\end{tabular}

Note: $\mathrm{N}=$ number of samples; $J_{n}, k$, and $Q_{n}$ are as defined in Table $1 ; J_{n}, k$, and $Q_{n}$ are geometric mean values.

TABLE 2

Alternating Field Demagnetization

\begin{tabular}{|c|c|c|c|c|c|c|c|c|c|c|c|c|c|c|c|c|c|c|c|c|c|}
\hline \multirow[b]{2}{*}{$\begin{array}{c}\text { Field } \\
\text { (peak oe) }\end{array}$} & \multicolumn{3}{|c|}{$332 \mathrm{~B}-35-2,33-36$} & \multicolumn{3}{|c|}{$332 \mathrm{~B}-36-2,59-61$} & \multicolumn{3}{|c|}{$334-20-1,114-116$} & \multicolumn{3}{|c|}{$335-6-1,99-101$} & \multicolumn{3}{|c|}{$335-6-1,105-107$} & \multicolumn{3}{|c|}{$335-6-1,112-114$} & \multicolumn{3}{|c|}{$335-13-3,100-111$} \\
\hline & $D$ & $I$ & $J / J_{o}$ & $D$ & $I$ & $J / J_{O}$ & $D$ & $I$ & $J / J_{o}$ & $D$ & I & $J / J_{o}$ & $D$ & $I$ & $J / J_{O}$ & $D$ & $I$ & $J / J_{O}$ & $D$ & $I$ & $J / J_{O}$ \\
\hline NRM & 225 & +7 & 1.00 & 211 & +27 & 1.00 & 297 & +37 & 1.00 & 255 & -58 & 1.00 & 256 & -67 & 1.00 & 247 & -68 & 1.00 & 204 & -73 & 1.00 \\
\hline 50 & 249 & -15 & 0.85 & 211 & +27 & 1.00 & 296 & +40 & 1.02 & 254 & -58 & 1.00 & 258 & -67 & 0.98 & 247 & -68 & 0.98 & 204 & -73 & 1.00 \\
\hline 100 & 269 & -38 & 0.68 & 211 & +27 & 0.95 & 295 & +42 & 0.74 & 255 & -58 & 0.97 & 259 & -67 & 0.95 & 248 & -68 & 0.92 & 204 & -73 & 0.99 \\
\hline 150 & 275 & -40 & 0.53 & 211 & +28 & 0.78 & 293 & +44 & 0.50 & 256 & -57 & 0.88 & 260 & -67 & 0.90 & 249 & -67 & 0.85 & 204 & -73 & 0.95 \\
\hline 200 & 280 & -42 & 0.43 & 210 & +29 & 0.56 & 293 & +43 & 0.38 & 257 & -57 & 0.77 & 260 & -66 & 0.80 & 250 & -67 & 0.75 & 204 & -73 & 0.87 \\
\hline 250 & 279 & -44 & 0.34 & 210 & +29 & 0.38 & 294 & +45 & 0.31 & 257 & -57 & 0.67 & 260 & -66 & 0.69 & 251 & -67 & 0.64 & 203 & -73 & 0.77 \\
\hline 300 & 278 & -45 & 0.29 & 209 & +29 & 0.26 & 292 & +42 & 0.25 & 257 & -58 & 0.53 & 260 & -67 & 0.53 & 251 & -67 & 0.56 & 206 & -72 & 0.66 \\
\hline 400 & 280 & -43 & 0.18 & 202 & +31 & 0.13 & 295 & +50 & 0.17 & 258 & -58 & 0.36 & 259 & -63 & 0.41 & 254 & -67 & $0 ; 42$ & 210 & -72 & 0.49 \\
\hline 600 & 274 & -46 & 0.11 & 218 & +34 & 0.07 & 290 & +37 & 0.11 & 260 & -60 & 0.17 & 264 & -65 & 0.18 & 242 & -72 & 0.23 & 182 & -74 & 0.26 \\
\hline 900 & 301 & -55 & 0.06 & 113 & +42 & 0.03 & 299 & +34 & 0.10 & 261 & -46 & 0.12 & 272 & -76 & 0.13 & 255 & -64 & 0.15 & 242 & -56 & 0.13 \\
\hline
\end{tabular}

Note: $D=$ Declination; $I=$ Inclination; $J / J_{O}=$ Normalized intensity.
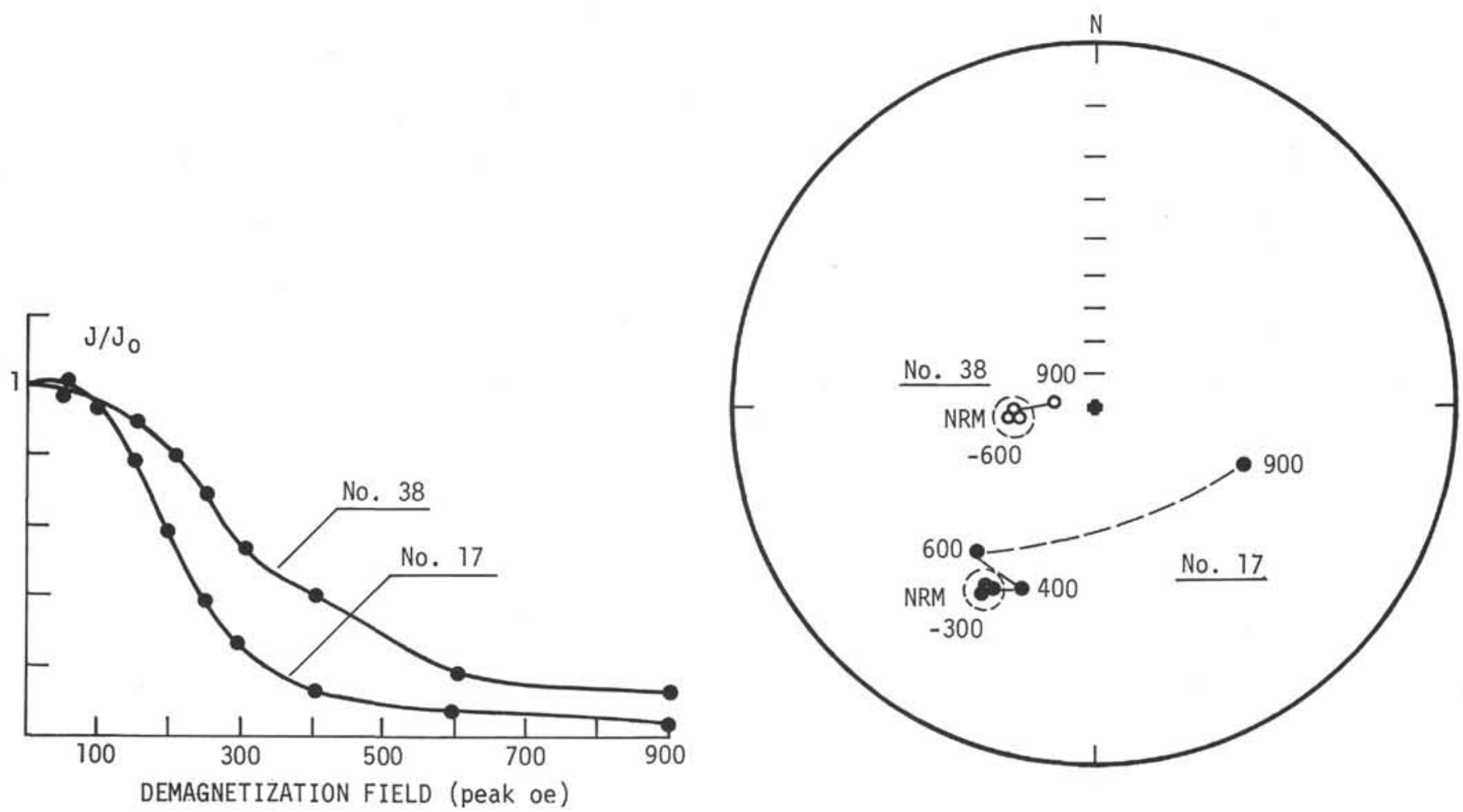

Figure 1. Samples $17(332 B-36-2,59-61 \mathrm{~cm})$ and $38(355-6-1,105-107 \mathrm{~cm})$. Normalized remanence versus AF demagnetizing field and the corresponding directional changes plotted on a stereographic (Wulff's) projection with the circumfererence as horizontal. Open and closed dots represent negative (north pole upwards) and positive (downwards) directions, respectively.

1), making a primary origin (Question 2) more likely. The low inclination affecting both polarities (Question
3) could be explained by an overall southward geological tilt, but then a column at least 275 meters 
TABLE 3A

Stepwise Thermal Demagnetization

\begin{tabular}{|c|c|c|c|c|c|c|c|c|c|c|c|c|c|c|c|}
\hline \multirow{2}{*}{$\begin{array}{c}\text { Temperature } \\
\left({ }^{\circ} \mathrm{C}\right)\end{array}$} & \multicolumn{3}{|c|}{$332 \mathrm{~B}-35-2,91-94$} & \multicolumn{3}{|c|}{$332 \mathrm{~B}-36-2,3-12$} & \multicolumn{3}{|c|}{$334-20-1,114-116$} & \multicolumn{3}{|c|}{$335-6-1,75-77$} & \multicolumn{3}{|c|}{$335-13-3,100-111$} \\
\hline & $D$ & $I$ & $J / J_{20}$ & $D$ & $I$ & $J / J_{20}$ & $D$ & $I$ & $J / J_{20}$ & $D$ & $I$ & $J / J_{20}$ & $D$ & $I$ & $J / J_{20}$ \\
\hline NRM & 304 & -33 & 1.00 & 64 & +16 & 1.00 & 289 & +24 & 1.00 & 259 & -58 & 1,00 & 208 & -73 & 1.00 \\
\hline & & -33 & 0 & 64 & +17 & 0.99 & 29 & +24 & & & -60 & 0. & 06 & -73 & 0.99 \\
\hline 10 & & -35 & 0 & 65 & +16 & 0.97 & 293 & +27 & & 58 & -60 & 7 & 205 & -73 & 0.97 \\
\hline & & -37 & 0.8 & 65 & +16 & 0.95 & 294 & +32 & & 58 & -60 & & & -73 & 0.95 \\
\hline & & -37 & 0.8 & 65 & +16 & 0. & 294 & +32 & & 59 & -59 & & & -72 & 0.92 \\
\hline & & -37 & & 65 & +14 & & 29 & +37 & & 58 & -60 & & & -73 & 0.89 \\
\hline & & -39 & $\mathrm{c}$ & 65 & +1 & & 296 & +40 & & 88 & -59 & & 207 & -73 & 0.92 \\
\hline & 3 & -41 & 0 & 65 & +10 & & 293 & +47 & 0. & 258 & -60 & & 205 & -73 & 0.66 \\
\hline & 300 & -41 & 0.1 & 59 & +12 & $0.1-1-3-1$ & 318 & +53 & & 258 & -61 & & 204 & -73 & 0.44 \\
\hline & 301 & -36 & & 60 & +11 & & 328 & +51 & & 299 & -80 & & 213 & -65 & 0.12 \\
\hline & & +74 & 0.02 & 211 & -5 & & 354 & +44 & & 249 & +2 & & 53 & -15 & 0.02 \\
\hline 775 & 106 & -65 & 0.01 & 70 & +22 & 0.00 & 83 & -16 & 0.03 & 339 & +25 & 0.01 & 162 & +70 & 0.01 \\
\hline
\end{tabular}

TABLE 3B

Continuous Thermal Demagnetization

\begin{tabular}{ccccccccccccccccc}
\hline Temperature & \multicolumn{3}{c}{$332 \mathrm{~B}-17-1,22-24$} & \multicolumn{3}{c}{$332 \mathrm{~B}-27-2,112-114$} & \multicolumn{2}{c}{$332 \mathrm{~B}-35-1,104-107$} & \multicolumn{3}{c}{$332 \mathrm{~B}-36-3,46-48$} & \multicolumn{3}{c}{$332 \mathrm{~B}-36-4,67-69$} \\
$\left({ }^{\circ} \mathrm{C}\right)$ & $D$ & $I$ & $J / J_{20}$ & $D$ & $I$ & $J / J_{20}$ & $D$ & $I$ & $J / J_{2} 0$ & $D$ & $I$ & $J / J_{20}$ & $D$ & $I$ & $J / J_{20}$ \\
\hline NRM & 40 & 1 & 1.00 & 231 & 18 & 1.00 & 243 & -26 & 1.00 & 309 & 4 & 1.00 & 85 & -7 & 1.00 \\
50 & - & - & - & 231 & 19 & 1.07 & - & - & - & - & - & - & - & - & - \\
100 & 40 & 0 & 0.96 & 230 & 20 & 1.01 & 244 & -34 & 0.98 & 308 & 6 & 0.96 & 84 & -9 & 0.98 \\
150 & 40 & 1 & 0.85 & 230 & 22 & 0.86 & 243 & -36 & 0.89 & - & - & - & 85 & -8 & 0.90 \\
200 & 39 & 2 & 0.67 & 231 & 22 & 0.63 & 243 & -37 & 0.74 & 309 & 8 & 0.77 & 84 & -12 & 0.79 \\
250 & 40 & 1 & 0.51 & 230 & 25 & 0.46 & 243 & -41 & 0.61 & 309 & 6 & 0.66 & 84 & -7 & 0.66 \\
300 & 40 & 2 & 0.41 & 234 & 23 & 0.33 & 241 & -40 & 0.53 & 309 & 6 & 0.56 & 85 & -10 & 0.63 \\
350 & - & - & - & 237 & 28 & 0.19 & 239 & -40 & 0.43 & 310 & 6 & 0.47 & 85 & -11 & 0.37 \\
400 & 47 & -4 & 0.06 & 256 & 23 & 0.11 & - & - & - & 310 & 6 & 0.33 & - & - & - \\
450 & 49 & -2 & 0.03 & 277 & 17 & 0.09 & 226 & -41 & 0.19 & 311 & 6 & 0.18 & 88 & -8 & 0.08 \\
500 & 40 & 8 & 0.02 & 300 & 8 & 0.09 & - & - & - & 308 & 7 & 0.10 & 80 & 0 & 0.09 \\
550 & 11 & 14 & 0.01 & - & - & - & 180 & -14 & 0.02 & 304 & 3 & 0.03 & 70 & 2 & 0.05 \\
$\geqslant 600$ & - & - & - & - & - & - & 82 & -34 & 0.01 & - & - & - & 0 & 13 & 0.02 \\
& $335-6-1,83-85$ & $335-6-1,122-124$ & $335-10-1,33-35$ & $335-13-1.78-88$ & & \\
Temperature & $D$ & $I$ & $J / J_{20}$ & $D$ & $I$ & $J / J_{20}$ & $D$ & $I$ & $J / J_{20}$ & $D$ & $I$ & $J / J_{20} 0$ & & \\
NRM & 257 & -66 & 1.00 & 258 & -69 & 1.00 & 198 & -76 & 1.00 & 22 & -70 & 1.00 & & \\
100 & 261 & -65 & 1.07 & 259 & -69 & 1.00 & 191 & -76 & 1.14 & 11 & -68 & 0.92 & & \\
200 & 258 & -65 & 0.90 & 257 & -68 & 0.76 & 199 & -75 & 1.04 & 14 & -65 & 0.52 & & \\
300 & 260 & -62 & 0.43 & 268 & -64 & 0.44 & 196 & -72 & 0.61 & 23 & -65 & 0.25 & & \\
400 & 255 & -67 & 0.15 & 323 & -71 & 0.09 & 193 & -78 & 0.17 & 27 & -60 & 0.11 & & \\
500 & 34 & -78 & 0.10 & 22 & -33 & 0.06 & 79 & -68 & 0.04 & 18 & -60 & 0.10 & & \\
550 & 17 & -71 & 0.12 & 22 & -19 & 0.08 & 56 & -17 & 0.03 & 23 & -65 & 0.06 & & \\
$\geqslant 600$ & 42 & -46 & 0.04 & 12 & 38 & 0.03 & - & - & - & 1 & -50 & 0.02 & & \\
\hline
\end{tabular}

Note: $D=$ Declination; $I=$ Inclination; $J / J_{20}=$ Normalized intensity.

thick would have had to be tilted. Furthermore, an important conclusion is that a magnetic contribution is needed from rock deeper in Sublayer 2B and perhaps Layer 3 to produce the observed surface anomaly. Provided Hole 332B is magnetically representative of Layer 2 rock elsewhere, this is an argument against "thin-layer" models that place the effective anomaly source into the uppermost part of Layer 2 (e.g., Talwani et al., 1971; Irving et al., 1970).

\section{SITE 335}

By comparison, 16 samples from a 70-meter section all have reverse polarity (Chapter 5 , this volume). Eleven of these $(6-1,53-55 \mathrm{~cm}$ to $6-1,129-131 \mathrm{~cm})$ are from a $78-\mathrm{cm}$ thick block showing well-aligned NRM directions with $I=-59^{\circ}$ to $-70^{\circ}$. All samples subjected to demagnetizations showed great stability in alter- nating fields to 600 oe (Table 2; Figure 1) and temperatures to $500^{\circ} \mathrm{C}$ (Tables $3 \mathrm{~A}, 3 \mathrm{~B}$; Figures 2, 3). The steep inclinations and their consistency with depth in these older basalts conflict with the results from Hole $332 \mathrm{~B}$.

\section{ACKNOWLEDGMENTS}

We are grateful to the organizers of DSDP for providing the samples. This work was supported by the National Research Council of Canada through Operating Grant A1946 to E.R. Deutsch and a DAGS Grant to G.S. Murthy.

\section{REFERENCES}

Deutsch, E.R. and Pätzold, R.R., 1976. Magnetic properties and domain state of basalt cores from the Nazca plate. In Yeats, R.S., Hart, S.R., et al., Initial Reports of the Deep Sea Drilling Project, Volume 34: Washington (U.S. Government Printing Office), p. 501-512. 

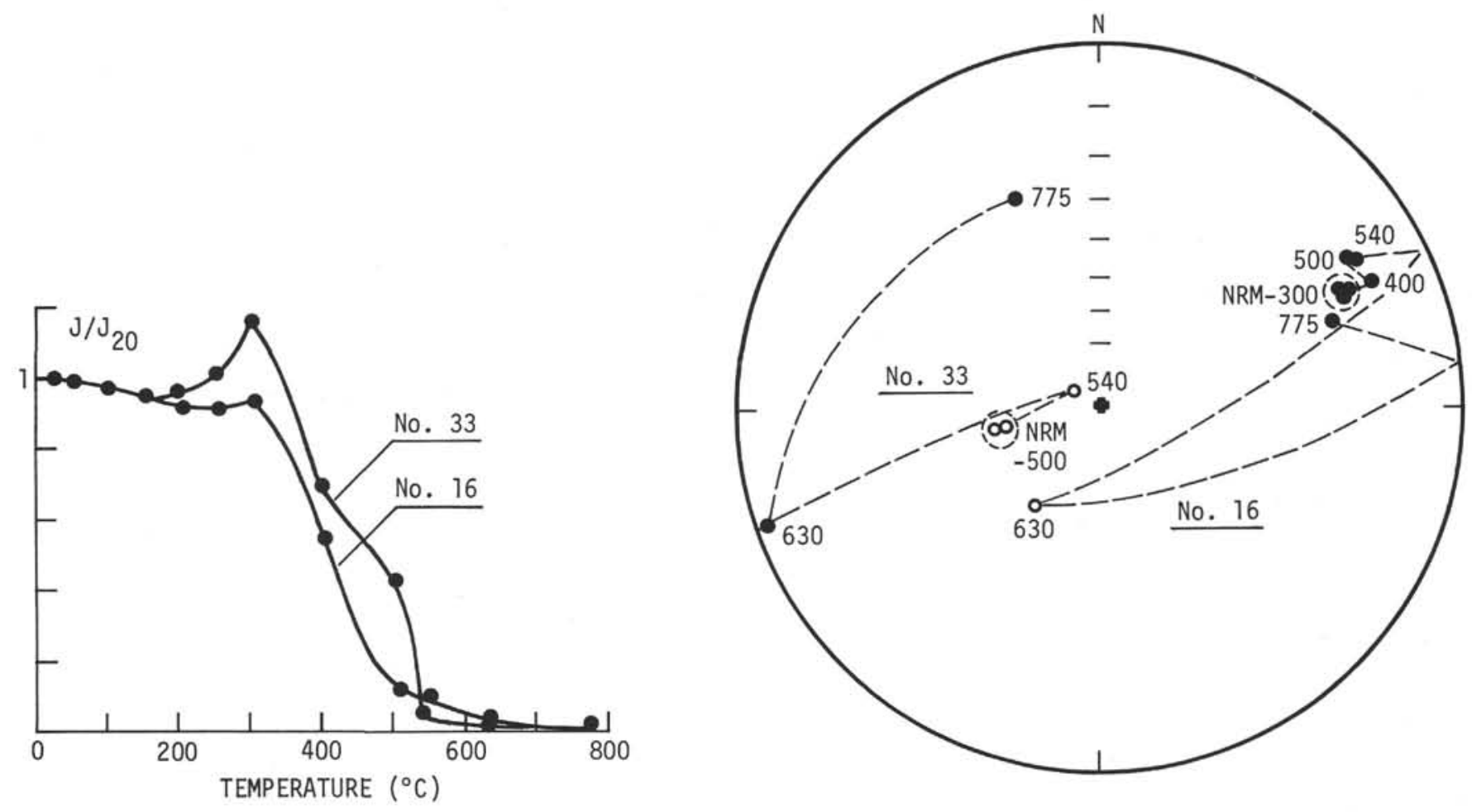

Figure 2. Samples $16(332 B-36-2,3-12 \mathrm{~cm})$ and $33(335-6-1,75-77 \mathrm{~cm})$. Normalized remanence versus temperature from stepwise thermal demagnetizations, and the directional changes plotted on a Wulff net. Other notations as in Figure 1. The cause of the peaks at $300^{\circ} \mathrm{C}$ is probably experimental.
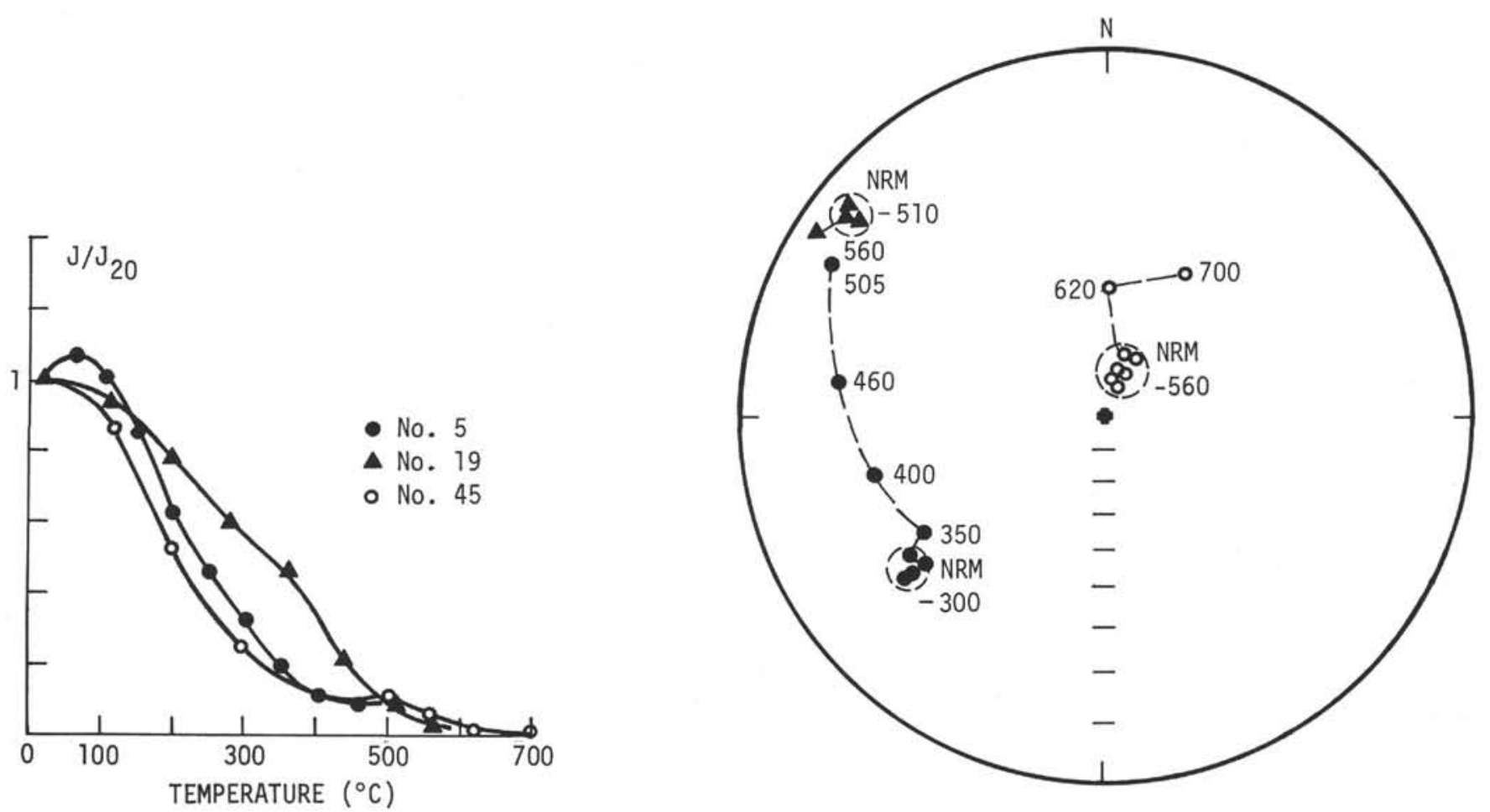

Figure 3. Left. Normalized remanence versus temperature from continuous thermal demagnetization experiments for Samples $5(332 B-27-2,112-114 \mathrm{~cm}), 19(332 B-36-3,46-48 \mathrm{~cm})$, and $45(335-13-1,78-88 \mathrm{~cm})$. Right. The corresponding directional changes plotted on a Wulff's net. 
Irving, E., Park, J.K., Haggerty, S.E., Aumento, F., and Loncarevic, B., 1970. Magnetism and opaque mineralogy of basalts from the mid-Atlantic ridge at $45^{\circ} \mathrm{N}$ : Nature, v. 228 , p. $974-976$.

JOIDES Scientific Staff, 1974. Deep sea drilling project, Leg 37-the volcanic layer: Geotimes, v. 19, Part 12, p. 16-18.
Lowrie, W., 1974. Oceanic basalt magnetic properties and the Vine and Matthews hypothesis: J. Geophys./Zeits. Geophys., v. 40, p. 513-536.

Talwani, M., Windisch, C.C., and Langseth, M.G., Jr., 1971. Reykjanes ridge crest: a detailed geophysical study: J. Geophys Res., v. 76, p. 473-517. 